\section{3-Jahres-Erdnuss-SLIT funktioniert und ist sicher}

\author{
Für die erste multizentrische Studie mit einer Erdnuss-SLIT liegen nun \\ die 3-Jahres-Langzeitdaten vor. Die Sicherheit war sehr gut, die \\ Langzeitwirksamkeit aber nicht überzeugend. Viele Patienten schafften \\ es nicht, regelmäßig ihre Tropfen zu nehmen.
}

\begin{abstract}
—ür Erdnussallergiker bietet die sublinguale Immuntherapie (SLIT) mit Erdnussallergenen einen sicheren Weg, teilweise oder vollständig ihre Symptome loszuwerden. In einer ersten randomisierten, doppelblinden und placebokontrollierten US-amerikanischen Multicenterstudie wurden nach einer 44-wöchigen Behandlung $70 \%$ der Verumpatienten (14/20) als Responder eingestuft, das heißt, sie vertrugen entweder $5 \mathrm{~g}$ oder die - zehnfache Menge Erdnusspulver im Ver운 gleich zum Studienbeginn symptomlos. In der Placebogruppe traf das auf $15 \%$ (3/20) zu. Die Sicherheit war sehr gut. In der dann offen weiter geführten $\circlearrowleft$ Phase erhielten alle Studienteilnehmer
\end{abstract}

acht Wochen nach Beendigung der SLIT noch vorhanden. Bei den klinischen Respondern im Studienjahr 2 stellte das Ärzteteam zusätzlich eine verminderte immunologische Aktivität bei Tests zur erdnussspezifischen Basophilenaktivierung sowie beim Prick-Schwellenwert fest.

eine Erhaltungsdosis von einmal täglich 165-1.386 $\mu \mathrm{g}$ Erdnussprotein in wässriger Lösung über einen Zeitraum von bis zu drei Jahren. Rund 98\% der Einzeldosen wurden gut vertragen, es traten keine Nebenwirkungen jenseits des Rachens oder schwere systemische Nebenwirkungen auf. Über $50 \%$ der Patienten beendeten die Behandlung vorzeitig, unter anderem aufgrund von Schwierigkeiten, die SLIT-Tropfen über drei Jahre regelmäßig, also täglich, einzunehmen. $\mathrm{Zu}$ Studienende waren vier der 35 Teilnehmer $(10,8 \%)$ vollständig desensibilisiert - sie vertrugen im oralen Provokationstest $10 \mathrm{~g}$ Erdnüsse. Dieser Effekt war bei diesen vier Studienteilnehmern auch
Fazit: Eine Erdnuss-SLIT über drei Jahre führte bei rund $10 \%$ der Erdnuss-Allergiker zu einer vollständigen Desensibilisierung, bei vielen weiteren Patienten zumindest zu einer moderaten Zunahme der Menge vertragener Erdnüsse. Rund $50 \%$ der Patienten beendeten die Studie allerdings vorzeitig. Das Konzept Nahrungsmittelallergen-SLIT funktioniert also, gearbeitet werden sollte jetzt vor allem an der Verbesserung der Therapieadhärenz. Dr. Barbara Kreutzkamp

Burks AW et al. Sublingual immunotherapy for peanut allergy: Longterm follow-up of a randomized multicenter trial. J Allergy Clin Immunol 2015; 135: 1240-8

\title{
Biozid Triclosan wirkt immunmodulierend
}

\section{Die Substanz Triclosan verstärkt im Tierversuch allergische Reaktionen. Zudem gibt es einen Zusammenhang zwischen dem Nachweis des Bakteriostatikums im Urin und Heuschnupfen. Unklar war bisher, wie es in die immunologischen Abläufe eingreift.}

D as zur Desinfektion in Kliniken und Praxen seit Langem genutzte Triclosan stand bisher vor allem wegen möglicher Resistenzentwicklungen bei übermäßiger Anwendung im Fokus. Inzwischen gibt es viele Hinweise aus Tiermodellen darauf, dass das Biozid bei Exposition auf der Haut das Immunsystem stimuliert. So erhöhte sich in den Versuchen die Zahl der B- und T-Zellen sowie der dendritischen und natürlichen Killerzellen in den nächstliegenden Lymphknoten.

Um darüber hinaus die Effekte von Triclosan genauer zu untersuchen, setzten Immunologen aus den USA bei Mäusen Haut und Lymphknoten einer bis zu 3\%igen Lösung aus und prüften den Effekt in vitro auch an menschlichem epidermalem Gewebe. Im Tierversuch wurde die Substanz alle 24 Stunden bis zu zwölf Tage lang in unterschiedlichen Konzentrationen (0,75-3\%) auf die Ohrmuschel aufgetragen.

Durch die Triclosanexposition kam es zu einer verstärkten Expression von TSLP („thymic stromal lymphopoietin“), IL-1- $\beta$ und TNF- $\alpha$ in der Haut. Dagegen wurden geringere Mengen an IL-25, IL-33 und IL-1- $\alpha$ synthetisiert. Im humanen Hautmodell kam es bei den Zytokinen TSLP, IL-1B und IL-33 zu ähnlichen Effekten. Auch auf zellulärer Ebene gab es Veränderungen nach der topischen Applikation von Triclosan. In den drainierenden Lymphknoten waren sowohl B-Lymphozyten (CD86- und GL-7-positiv) und dendritische Zellen (CD80- und CD86-positiv) als auch T2-Helferzellen und CD4-TLymphozyten vermehrt nachweisbar. Alle Effekte traten auch dann auf, wenn es nach der Triclosanbehandlung nicht zu Hautirritationen kam.

Eine besondere Rolle bei der Th2-vermittelten allergischen Reaktion auf Triclosan spielt offenbar das Zytokin TSLP. In den Tierversuchen sank durch die Behandlung mit einem Antikörper gegen TSLP in den Lymphknoten vor allem die Zahl der B-Zellen und der dendritischen Zellen. Auch die Zahl CD44-positiver THelferzellen (CD4-positiv) nahm durch die Antikörpertherapie signifikant ab.

Fazit: Die topische Anwendung von Triclosan hat messbare immunmodulatorische Effekte, und dies auch bei fehlender Hautirritation. Dies ist eine wichtige Erkenntnis, zumal die Substanz in verschiedenen Produkten in einer Konzentration von $0,1-1 \%$ enthalten ist. Die im Gesundheitswesen sinnvolle Anwendung des Biozids muss mit dem potenziellen Risiko durch seine immunmodulatorische Wirkung abgewogen werden. Peter Leiner

Marshall NB et al. Triclosan Induces Thymic Stromal Lymphopoietin in Skin Promoting Th2 Allergic Responses. Toxicol Sci 2015; 147: 127-39 